DRAFT VERSION DECEMBER 30, 2016

Preprint typeset using LATEX style AASTeX6 v. 1.0

\title{
THERMAL DISK WINDS IN X-RAY BINARIES: REALISTIC HEATING AND COOLING RATES GIVE RISE TO SLOW, BUT MASSIVE OUTFLOWS
}

\author{
N. Higginbottom ${ }^{1}$, D. ProgA ${ }^{2}$, C. KNIGGE ${ }^{1}$, AND K. S. LONG $^{3,4}$
}

\footnotetext{
${ }^{1}$ School of Physics and Astronomy, University of Southampton, Highfield, Southampton, SO17 1BJ, UK

${ }^{2}$ Department of Physics \& Astronomy, University of Nevada, Las Vegas, 4505 S. Maryland Pkwy, Las Vegas, NV 89154-4002, USA

${ }^{3}$ Space Telescope Science Institute, 3700 San Martin Drive, Baltimore, MD, 21218, USA

${ }^{4}$ Eureka Scientific Inc., 2542 Delmar Avenue, Suite 100, Oakland, CA, 94602-3017, USA
}

\begin{abstract}
A number of X-ray binaries exhibit clear evidence for the presence of disk winds in the high/soft state. A promising driving mechanism for these outflows is mass loss driven by the thermal expansion of X-ray heated material in the outer disk atmosphere. Higginbottom \& Proga recently demonstrated that the properties of thermally-driven winds depend critically on the shape of the thermal equilibrium curve, since this determines the thermal stability of the irradiated material. For a given spectral energy distribution, the thermal equilibrium curve depends on exact balance between the various heating and cooling mechanisms at work. Most previous work on thermally-driven disk winds relied an analytical approximation to these rates. Here, we use the photoionization code CLOUDY to generate realistic heating and cooling rates which we then use in a $2.5 \mathrm{D}$ hydrodynamic model computed in ZEUS to simulate thermal winds in a typical black-hole X-ray binary. We find that these heating and cooling rates produce a significantly more complex thermal equilibrium curve, with dramatically different stability properties. The resulting flow, calculated in the optically thin limit, is qualitatively different from flows calculated using approximate analytical rates. Specifically, our thermal disk wind is much denser and slower, with a mass-loss rate that is a factor of two higher and characteristic velocities that are a factor of three lower. The low velocity of the flow $-v_{\max } \simeq 200 \mathrm{~km} \mathrm{~s}^{-1}-$ may be difficult to reconcile with observations. However, the high mass-loss rate $-15 \times$ the accretion rate - is promising, since it has the potential to destabilize the disk. Thermally-driven disk winds may therefore provide a mechanism for state changes.
\end{abstract}

Keywords: accretion, accretion disks - hydrodynamics - methods: numerical - X-rays: binaries

\section{INTRODUCTION}

Disk winds are seen in a range of accreting objects, from cataclysmic variables (CVs) to active galactic nuclei (AGN). The existence of these outflows is inferred from the presence of blue-shifted absorption features, and detailed analysis of these features can provide information regarding the ionization state, geometry and kinematics of the flow.

Amongst the many classes of objects exhibiting such blue shifted absorption lines are X-ray binaries (XRBs). These are thought to be systems in which a neutron star (NS) or black hole $(\mathrm{BH})$ is accreting material from a companion star. XRBs are further subdivided into two classes. In high-mass XRBs (HMXBs), the compact object has a massive companion and often accretes material from the wind of this early-type star. By contrast, low-mass XRBs (LMXBs) contain late-type secondaries, and accretion is always driven by Roche-lobe overflow from these donors. Our focus here is on LMXBs.

LMXBs are highly variable, exhibiting dramatic changes in their spectral energy distribution (SED). Sometimes they exhibit a blackbody disk-like spectrum (referred to as the 'high/soft' state), and sometimes the SED is dominated by a hard power-law spectrum (the 'low/hard' state). This second state is often accompanied by a radio jet which disappears as the source transitions into the high/soft state (Fender et al. 2004). The X-ray signatures of highly ionized gas have been observed in more than a dozen LMXBs (Díaz Trigo \& Boirin 2016, and refs therein), with 8 showing evidence of an outflow. Outflows are always seen in systems in the high/soft state that are viewed close to edge-on(Ponti et al. 2012). The most likely candidate for this outflowing gas is an equatorial wind arising from the accretion disk, although the mechanism for launching and accelerating such XRB winds is still a subject of active research.

One promising possibility is that the wind is thermally driven, with the upper surface of the accretion disk puffing up as it is heated by X-rays from the inner disk and corona. Expansion of this gas as it is further heated then accelerates the material away from the central object as a wind. Such winds are expected to arise at fairly large distances from the central object, where the local isothermal sound speed at the Compton temperature exceeds the local escape velocity (Begelman et al. 1983; Woods et al. 1996). Alternative wind-driving mechanisms include acceleration via magnetocentrifugal forces (e.g. Blandford \& Payne 1982; Emmering et al. 1992; Miller et al. 2006; Chakravorty et al. 2016) and radiation pressure acting on electrons or spectral lines (e.g. 
Icke 1980; Shlosman \& Vitello 1993; Shlosman et al. 1985; Proga \& Kallman 2002).

In order to test these models, we must rely upon measurements of the kinematics of the wind from spectral features. The observationally inferred wind velocities are typcially between about $400 \mathrm{~km} \mathrm{~s}^{-1}$ and $3000 \mathrm{~km} \mathrm{~s}^{-1}$ across both BH and NS systems (Díaz Trigo \& Boirin 2016), although one system does show a marginal detection of higher velocity gas (King et al. 2012). Photoionization modelling can also provide estimates of the column density of the absorbing gas, along with its ionization parameter. The inferred columns lie between about $5 \times 10^{20}$ and $10^{24} \mathrm{~cm}^{-2}$ (Díaz Trigo \& Boirin 2016), with the plasma in a highly ionized state $(\log \xi \geq 3)$. The ionization parameter $\xi$ is defined here as $\xi=L_{X} / n r^{2}$ where $L_{X}$ is the X-ray luminosity, $r$ is the distance between the gas and the X-ray source, and $n$ is the gas density.

Focusing on thermally driven disk winds, Higginbottom \& Proga (2015, hereafter HP15) took a hydrodynamical model of such an outflow first developed by Luketic et al. (2010, hereafter L10) and investigated its sensitivity to changes in the assumed heating and cooling rates. They found that a reduction in line cooling (which could perhaps reflect optical depth effects) and an increase in photoionization heating (which could perhaps mimic SED effects) could significantly increase the mass-loss rate via the thermal wind, from about 4 times the accretion rate to over 40 times. Such a high massloss rate would be expected to destabilise the disk and could perhaps supply a mechanism for state change (Shields et al. 1986). In addition, the velocities of the outflowing gas approached those inferred from X-ray observations.

These results demonstrated that heating and cooling rates are critical in the launching and acceleration of thermally driven disk winds. However, the rates used in L10 were analytical approximations to more complex underlying rates, and the HP15 simply added multiplicative factors to these approximations. Given the sensitivity of the outflow properties to heating and cooling found in HP15, we decided to carry out a new set of simulations with more detailed and realistic heating and cooling rates. We continue to use the hydrodynamics code ZEUS (Stone \& Norman 1992), extended by (Proga et al. 2000), but now we use the radiative transfer and ionization code CLOUDY (Ferland et al. 2013) to compute heating and cooling rates for the model. This work is part of an ongoing push towards full radiation hydrodynamic simulations of thermally-driven disk winds that will be able to take full account of frequency dependant optical depth effects.

\section{METHOD}

Radiative heating enters the equations of hydrodynamics via the energy conservation equation

$$
\rho \frac{D}{D t}\left(\frac{e}{\rho}\right)=-P \nabla \cdot \mathbf{v}+\rho \mathcal{L},
$$

where $\mathcal{L}$ is the net heating rate per unit mass e is the internal energy density, and $\mathrm{v}$ is the velocity. The first term on the right hand side of the equation represents adiabatic heating/cooling, whilst the second term represents the radiative heating/cooling. Thermal conduction (TC) can also be an important process in setting the thermal balance in X-ray coronae (e.g. Jimenez-Garate et al. 2001). Therefore, we computed the TC contribution to the gas heating/cooling using our solution and compared this contribution with all terms in Eq. 1. We found that in the thermal wind part of our solution, the conduction is negligible compared to adiabatic cooling and radiative heating. As expected the conduction rate reaches a maximum where the temperature increases most rapidly but even there the conduction rate is two order of magnitude lower than the adiabatic rate. This comparison indicates that $\mathrm{TC}$ has a small effect on the X-ray winds.

In the optically thin limit, for a given illuminating SED, $\mathcal{L}$ is a function of plasma temperature $\mathrm{T}$, and ionization parameter $\xi$, defined here as

$$
\xi=\frac{L_{x}}{n_{H} r^{2}},
$$

where $L_{X}$ is the X-ray luminosity (integrated from $13.6 \mathrm{eV}$ to infinity), $n_{H}$ is the Hydrogen number density of the gas, and $r$ is the distance between the source of ionizing flux and the gas. Note that this definition is slightly different to that used in HP15. This rescaling is motivated by the aims of our current project, in which we aim to use heating and cooling rates calculated directly from photoionization codes rather than from the parametrised equations calculated by (Blondin 1994, hereafter B94) which were used in HP15. Since the ionization parameter is defined in both XSTAR and CLOUDY as a ratio between the ionizing radiation (either energy or photon flux) and the Hydrogen density, choosing this definition of $\xi$ simplifies comparisons between the codes.

The heating and cooling rates parametrised by the 'Blondin equations' from B94 include expressions for net Compton heating $\left(G_{\text {Compton }}\right)$ and bremsstrahlung cooling $\left(L_{b}\right)$ both derived from first principles, and X-ray heating $\left(G_{X}\right)$ and line cooling $\left(L_{l}\right)$, which are fits to rates calculated from XSTAR simulations (Blondin et al. 1990). Line cooling represents the energy lost from the plasma to the photon field due to boundbound and free-bound interactions, whilst X-ray heating represents heating caused by bound-free (photoionization) interactions. These equations enter Equation 1 via

$$
\rho \mathcal{L}=n_{H} n_{e}\left(G_{\text {Compton }}+G_{X}-L_{l}-L_{b}\right) .
$$

Whilst the Blondin equations provide reasonable estimates of the heating and cooling rates, particularly for Compton and bremsstrahlung effects, the line cooling and X-ray heating rates are fits to more complex underlying data. Using these equations is computationally efficient. However, here we are interested in seeing how the details of the heating and cooling rates affect the solutions. In addition, the simulations used to generate the fits are some 25 years old, and it is worth updating the simulations with improved and more complete atomic data and codes. To achieve this, we compute heating and cooling rates in CLOUDY from a model consisting of a thin shell of plasma surrounding a point-like central radiation source. We vary the ionization parameter and temperature in the shell in order to produce a grid of heating and cooling rates which are supplied to ZEUS via look-up tables. 
These are used to obtain $\mathcal{L}$ at each hydrodynamic time step via bilinear interpolation in $\log (\xi, \mathrm{T})$ space.

In order to compare with the simulations presented by HP15, we use the same SED that was used to compute the Blondin heating and cooling rates. ${ }^{1}$ B94 used XSTAR to compute these rates, and the adopted SED was a Bremsstrahlung spectrum of the form

$$
f_{\nu} \sim \exp \left(-h \nu / k_{B} T_{X}\right)
$$

The net heating and cooling rates include all heating and cooling mechanisms included in version 13 of CLOUDY, with the exception of induced Compton heating, which we remove.This mechanism would not be expected to be important in a real system, and only appears in our thin shell model because the CLOUDY Bremsstrahlung SED does not have a lower frequency cut-off. This means that the radiation density exceeds that of a blackbody at low temperatures. As in HP15, we make the assumption that the wind is optically thin when computing $\xi$ in each cell.

The simulations are carried out on a spherical polar grid, running from $0^{\circ}$ to $90^{\circ}$ in polar angle $\left(90^{\circ}\right.$ representing the mid-plane) and from $R_{\text {in }}$ to $R_{\text {out }}$ in radius. In the radial dimension, the spacing between grid points increases with radius, such that $d r_{k+1} / d r_{k}=1.02$ thereby giving greater resolution in the inner parts of the flow. Conversely, going from the polar direction towards the disk, the angular grid spacing decreases, such that $d \theta_{k+1} / d \theta_{k}=0.95$. This gives more resolution near the disk, where we expect any flow will be accelerating, and therefore changing density, velocity and temperature quickly. A reflecting boundary condition (BC) is used along the midplane, outflow BCs at the inner and outer radial edges and a rotational $\mathrm{BC}$ along the axis of rotational symmetry. A density floor of $10^{-22} \mathrm{~g} \mathrm{~cm}^{-3}$ is applied throughout the model.

The initial state of the simulation is a hydrostatically supported disk, with the density along the mid-plane set by

$$
\rho(r)=\rho_{0}\left(\frac{r}{R_{I C}}\right)^{-2},
$$

where $R_{I C}$ is the Compton radius, i.e. the radius at which the local isothermal sound speed (at the Compton temperature, $\mathrm{T}_{\mathrm{IC}}$ ) is equal to the escape velocity,

$$
R_{I C}=\frac{G M_{B H} \mu m_{H}}{k_{B} T_{I C}} .
$$

Here, $M_{B H}$ is the mass of the central object and $\mu$ is the mean molecular mass (set to 0.6 in these simulations). $\mathrm{R}_{\mathrm{IC}}$ is the radius beyond which one would expect a thermal wind to arise. For a black hole mass of $7 M_{\odot}$, and for the Compton temperature $T_{I C}=1.4 \times 10^{7} \mathrm{~K}, \mathrm{R}_{\mathrm{IC}}=4.8 \times 10^{11} \mathrm{~cm}$.

The choice of the value of $\rho_{0}$ sets the ionization parameter at the midplane. In order to explain the significance of this choice, we briefly discuss the schematic stability curve shown in Figure 1. The familiar 's' shaped curve, formed by

\footnotetext{
${ }^{1}$ It should be noted that this method permits any SED to be used in calculating the heating and cooling rates, and so future simulations using observed SEDs are possible.
}

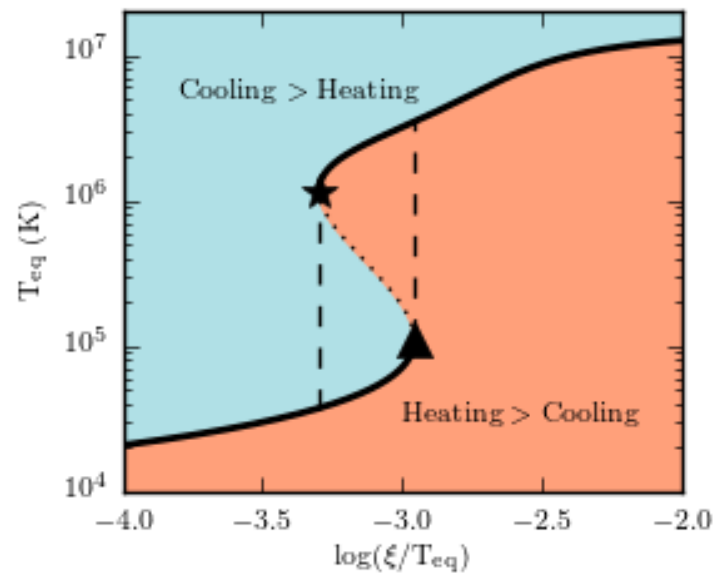

Figure 1. An schematic thermal equilibrium curve showing parts of the phase space where heating exceeds cooling and vice versa. The location of $\xi_{\text {cool,max }}$, the maximum ionization parameter on the cool stable branch is indicated by a triangle, and $\xi_{\text {hot,min }}$, the lowest ionization parameter on the hot stable branch is indicated by a star. The dotted part of the thermal equilibrium curve is unstable.

the two thick lines connected by the dotted line, represents the points on the phase diagram where heating is equal to cooling (the quantity $\xi / \mathrm{T}$ is inversely proportional to pressure). For gas in thermal equilibrium on either of the two thick lines, the equilibrium is stable. For example, if it is heated up in some way, it enters a part of the phase diagram where the cooling rate exceeds the heating rate, so it will cool back to the equilibrium state. Similarly, if gas is cooled away from from such locations on the thermal equilibrium curve, it finds itself in a regime where heating exceeds cooling, restoring the equilibrium. The situation is different for gas in thermal equilibrium on the dotted line. If such a parcel of gas is heated up, it moves into a region where heating exceeds cooling, so it will continue to heat up until it reaches the upper portion of thermal equilibrium curve - the hot stable branch. Likewise, if gas is cooled it will enter the part of the phase diagram where the cooling rate exceeds the heating rate. It will continue to cool until it reaches the cool stable branch. Thus the dotted part of the S-curve is thermally unstable.

In our simulations, we are interested in modelling outflowing gas arising from the surface of an accretion disk. Points lying on the cool stable branch are in thermal equilibrium at a relatively low temperature, and do not contribute to any outflow. They can be considered to be a part of a stable accretion disk atmosphere and we therefore wish to exclude such cells from our simulation. Maximum heat input to the gas is achieved for gas with an ionization parameter of $\xi_{\text {cool,max }}$, indicated by a triangle on Figure 1. Assuming that the gas is free to expand, this increase in internal energy will result in acceleration of the gas. Gas lying at this point which is heated up slightly has the maximum change in temperature and hence change in internal energy before reaching the upper stable branch. The temperature of the gas at $\xi_{\text {cool,max }}$, $\mathrm{T}_{\text {cool,max }}$ is therefore also important. The cooler the gas is at this point, the greater the potential heat input is, and therefore 


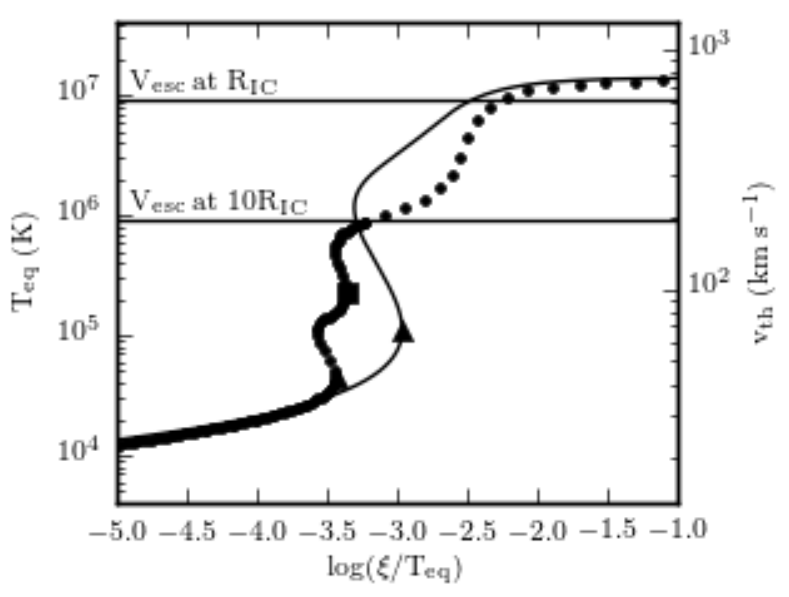

Figure 2. Stability curves computed from a thin shell CLOUDY model (dashed line) and that for the parametrised heating and cooling rates used in HP15 (solid line). The second y-axis shows the RMS thermal velocity for gas at these temperatures, and the two horizontal lines show the escape velocity at $1 \mathrm{R}_{\mathrm{IC}}$ and $10 \mathrm{R}_{\mathrm{IC}}$.

the greater the acceleration. In the absence of strong magnetic fields, this change takes place at constant pressure and the gas parcels will follow a vertical track on this diagram. We therefore set $\rho_{0}$ so that gas at the midplane has this value of $\xi$ and thus can be considered to be the uppermost thermally stable surface of the accretion disk atmosphere. The initial temperature for cells along the midplane is set according to the standard thin disk approximation (Shakura \& Sunyaev 1973) with other cells set to the same effective temperature as the midplane cell vertically below them.

We have carried out two new simulations. Firstly, we use the Blondin equations, with no prefactors applied to compute the heating and cooling rates - our baseline test. This is broadly comparable to Model A in HP15, and we refer to it as the 'Blondin run'. Secondly, we have carried out a simulation where the heating and cooling rates are set using the CLOUDY lookup table described above. We refer to this run as the 'Cloudy run'.

Figure 2 shows the stability curves for the Blondin equations, and the CLOUDY simulations. The location of $\xi_{\text {cool,max }}$ is indicated on each one by a triangle. It is clear that the shape of the CLOUDY curve is more complex ${ }^{2}$, showing an intermediate stable zone at a temperature of about $2 \times 10^{5}$ $\mathrm{K}$. Instability sets in once more at the location shown by a square. We also show the isothermal sound speed on the second axis and the escape velocity at 1 and $10 R_{\mathrm{IC}}$. We see that gas on the upper stable branch has a thermal velocity in excess of the escape velocity at $1 \mathrm{R}_{\mathrm{IC}}$ as expected.

Another important parameter is the temperature at which gas parcels heating up at constant pressure from $\xi_{\text {cool,max }}$ intersect with the stability curve once more, $\mathrm{T}_{\text {hot,min }}$. The difference between this and $\mathrm{T}_{\text {cool,max }}$ is a measure of how much energy can be absorbed by the gas as it heats up in the un-

\footnotetext{
${ }^{2}$ See Dyda et al. (2016) for the effect on the stability curve of a range of different SEDs.
}

\begin{tabular}{|c|c|c|c|c|}
\hline Physical Parameters & Blondin & $\begin{array}{l}\text { Extended } \\
\text { Blondin } \\
\end{array}$ & Cloudy & $\begin{array}{l}\text { Extended } \\
\text { Cloudy } \\
\end{array}$ \\
\hline $\mathrm{M}_{\mathrm{BH}}\left(\mathrm{M}_{\odot}\right)$ & 7 & 7 & 7 & 7 \\
\hline $\mathrm{T}_{\mathrm{X}}\left(10^{6} \mathrm{~K}\right)$ & 56 & 56 & 56 & 56 \\
\hline $\mathrm{L}_{\mathrm{X}}\left(10^{37}\right.$ ergs s$\left.^{-1}\right)$ & 3.3 & 3.3 & 3.3 & 3.3 \\
\hline $\log \left(\xi_{\text {cold,max }}\right)$ & 2.10 & 2.10 & 1.226 & 1.226 \\
\hline $\log \left(\Xi_{\text {cold,max }}\right)$ & 1.33 & 1.33 & 0.877 & 0.877 \\
\hline $\mathrm{T}_{\mathrm{eq}}\left(\xi_{\text {cold,max }}\right)\left(10^{3} \mathrm{~K}\right)$ & 111 & 111 & 43.7 & 43.7 \\
\hline$\rho_{0}\left(10^{-12} \mathrm{~g} \mathrm{~cm}^{-3}\right)$ & 2.75 & 2.75 & 20.3 & 20.3 \\
\hline $\mathrm{R}_{\mathrm{IC}}\left(10^{11} \mathrm{~cm}\right)$ & 4.82 & 4.82 & 4.82 & 4.82 \\
\hline \multicolumn{5}{|l|}{ Grid parameters } \\
\hline $\mathrm{R}_{\min }\left(10^{10} \mathrm{~cm}\right)$ & 2.4 & 2.4 & 2.4 & 2.4 \\
\hline $\mathrm{R}_{\max }\left(10^{10} \mathrm{~cm}\right)$ & 96 & 960 & 96 & 960 \\
\hline $\mathrm{R}_{\text {disk }}\left(10^{10} \mathrm{~cm}\right)$ & 96 & 96 & 96 & 96 \\
\hline $\mathrm{R}_{\text {ratio }}$ & 1.05 & 1.05 & 1.05 & 1.05 \\
\hline $\mathrm{N}_{\mathrm{R}}$ & 80 & 200 & 80 & 200 \\
\hline$\theta_{\min }$ & 0.0 & 0.0 & 0.0 & 0.0 \\
\hline$\theta_{\max }$ & 90.0 & 90.0 & 90.0 & 90.0 \\
\hline$\theta_{\text {ratio }}$ & 0.95 & 0.95 & 0.95 & 0.95 \\
\hline $\mathrm{N}_{\theta}$ & 100 & 100 & 100 & 100 \\
\hline \multicolumn{5}{|l|}{ Derived wind properties } \\
\hline \multicolumn{5}{|l|}{$\mathrm{V}_{\mathrm{r}}\left(\rho>1 \times 10^{12}\right)$} \\
\hline max, blueshifted $\left(\mathrm{km} \mathrm{s}^{-1}\right)$ & 79 & 78 & 21 & 19 \\
\hline $\mathrm{n}_{\mathrm{H}}\left(60^{\circ}\right)\left(10^{22} \mathrm{~cm}^{-2}\right)$ & 2.9 & 3.3 & 3.6 & 4.7 \\
\hline $\mathrm{n}_{\mathrm{H}}\left(80^{\circ}\right)\left(10^{22} \mathrm{~cm}^{-2}\right)$ & 16 & 17 & 62 & 63 \\
\hline$\dot{\mathrm{M}}_{\text {wind,outer }}\left(10^{18} \mathrm{~g} \mathrm{~s}^{-1}\right)$ & 3.4 & 3.6 & 2.3 & 6.7 \\
\hline$\dot{\mathrm{M}}_{\text {wind,outer }}\left(\dot{\mathrm{M}}_{\mathrm{acc}}\right)$ & 7.8 & 8.2 & 5.2 & 15 \\
\hline
\end{tabular}

Table 1. The heating and cooling parameters adopted in the simulations, and some key parameters of the resulting winds.

stable part of the stability plot. The fact that the value of $T_{\text {cool,max }}$, is lower for the Cloudy run would tend to suggest that more energy can be absorbed in this case, but the presence of the intermediate stable zone and the relatively low value of $\mathrm{T}_{\text {hot,min }}$ means that this is not the case.

In order to make the comparison a fair one, we set the midplane density in a comparable way for both runs. As in HP15, we choose to set the density such that the ionization parameter at the midplane is equal to $\xi_{\text {cool,max }} .{ }^{3}$ For the Blondin run, this is slightly different from the density used in HP15 because of the rescaling of $\xi$. This change results in an increase from $\rho_{0}=1.14 \times 10^{-12} \mathrm{~g} \mathrm{~cm}^{3}$ to $2.75 \times 10^{-12} \mathrm{~g} \mathrm{~cm}^{3}$. For the Cloudy run $\rho_{0}=2.03 \times 10^{-11} \mathrm{~g} \mathrm{~cm}^{-3}$. For all the runs we set $\mathrm{L}_{\mathrm{X}}=3.3 \times 10^{37} \mathrm{ergs} \mathrm{s}^{-1}$ which implies an accretion rate of $\dot{\mathrm{M}}_{\mathrm{acc}}=4.4 \times 10^{17} \mathrm{~g} \mathrm{~s}^{-1}$ (assuming an efficiency of $8.3 \%$ for $\left.\mathrm{M}_{\mathrm{BH}}=7 \mathrm{M}_{\odot}\right)$.

\section{RESULTS}

\footnotetext{
3 Note that since both the midplane density and flux drop off as $\mathrm{r}^{-2}$ the ionization parameter is constant in all midplane cells. This is not to say we suggest that the radial drop off in density is a realistic parametrisation of the density drop off at any given depth within the disk, rather that we assume that there will be some depth within the disk atmosphere at which the ionization parameter will be equal to $\xi_{\text {cool,max }}$. We would expect the denser disk photosphere to exist below this point.
} 

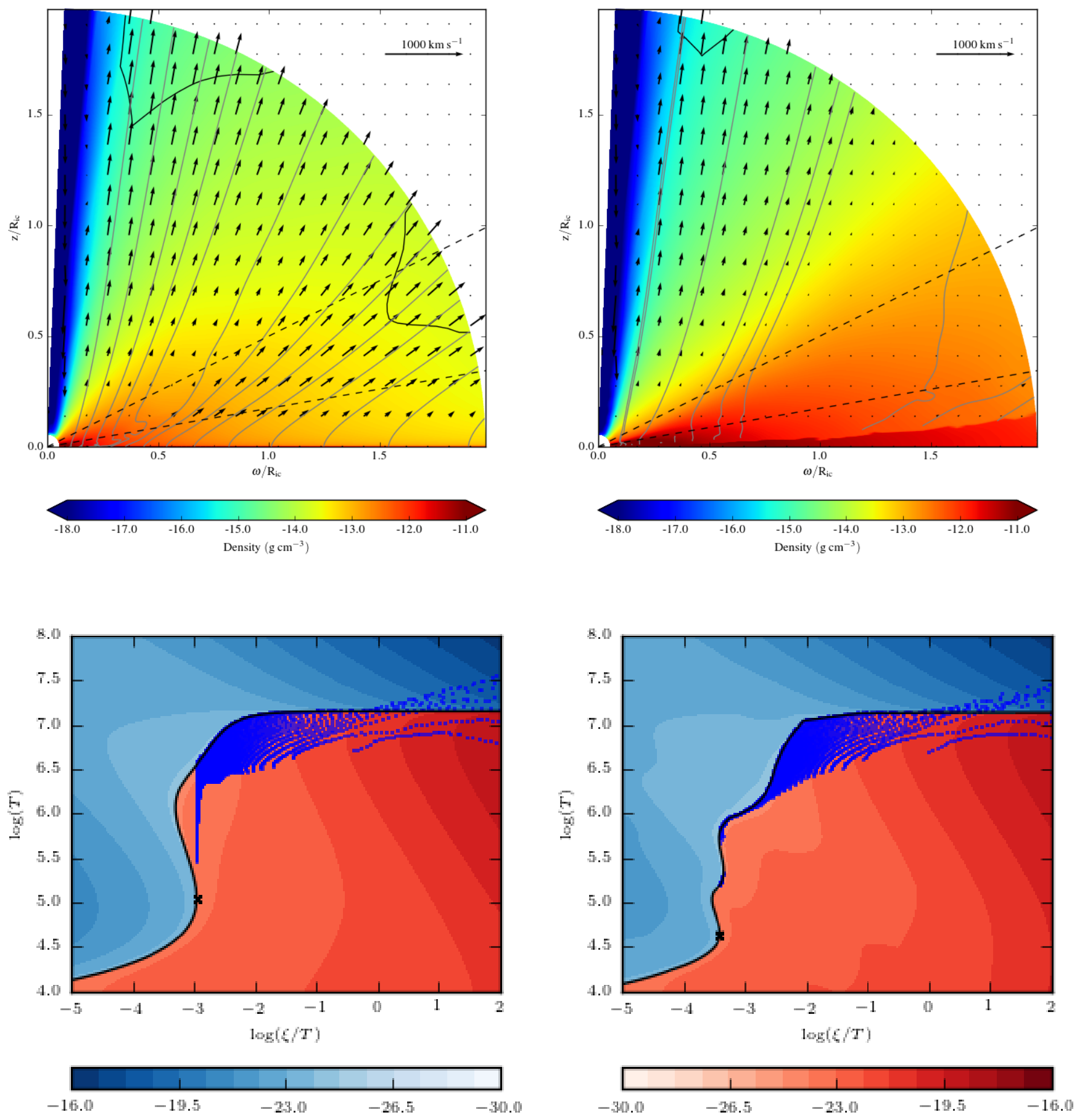

Figure 3. Comparison between two wind geometries. Left hand panels are for Blondin run, right hand panels for Cloudy run. Grey lines show streamlines, and the black line shows the location of the Mach1 surface (seen as two 'v' shaped features at the outer edge of the Blondin run, and only barely visible at the top of the Cloudy run). The two dotted lines show the location of the $60^{\circ}$ and $80^{\circ}$ sightlines The two lower panels show the location of all the cells in each model on a phase diagram. The contours show the net heating/cooling rate and the equilibrium curve is plotted as a thick black line. 
The top two panels of Figure 3 show the density and velocity structure of the Blondin run (left) compared to Cloudy run (right). It is immediately clear that the equatorial flow is much slower in the new run, although it is denser. We compute a mass outflow rate through the outer simulation boundary by performing the summation

$$
\dot{M}=\sum v_{r} \rho d A
$$

over all of the outer radial cells. As we would expect, the Blondin run has a very similar structure to model A in HP15, although the outflow rate is doubled to about $3.4 \times 10^{18} \mathrm{~g} \mathrm{~s}^{-1}$ $\left(8 \dot{\mathrm{M}}_{\mathrm{acc}}\right)$. This is because the midplane density in the new simulation is roughly twice that of the older run for the same value of $\xi$ because of the revised definition of $\xi$.

The Cloudy run has a somewhat variable outflow rate with time, failing to settle down to a steady state even after $10^{7}$ seconds of simulation time (which represents at least 32 sound crossing times, calculated by dividing the radial extent of the model by the slowest isothermal sound speed seen in any cell). The mean mass outflow rate over the last $10^{6} \mathrm{~s}$ is $2.3 \times 10^{18} \mathrm{~g} \mathrm{~s}^{-1}\left(5 \dot{\mathrm{M}}_{\mathrm{acc}}\right)$ with a standard deviation of $2.9 \times 10^{17} \mathrm{~g} \mathrm{~s}^{-1}$. This instability suggests that in this run our domain size may not be large enough to capture any stable outflow, and we are instead only simulating the turbulent 'corona' which exists in the original model, but only in the inner $0.5 \mathrm{R}_{\mathrm{IC}}$.

There is a clear density 'step' at around $85^{\circ}$ in the Cloudy run. This is caused by the intermediate stable zone on the stability curve. Previous studies have noted the presence of intermediate stable zones. For example one of the stability curves used by Nayakshin et al. (2000) had an intermediate zone which could 'interrupt' the heating of plasma from the cool stable branch to the hot stable branch. However an investigation of the effect on gas dynamics was beyond the scope of their study. The standard AGN SED used by Woods et al. (1996) also gave rise to a stability curve with an intermediate stable zone. In this case, because the intermediate unstable zone exists at a lower value of $\xi / \mathrm{T}$ than $\xi_{\text {cool,max }} / \mathrm{T}$, it is not possible for gas heating up from the cool branch to reach this area of the stability curve and so there is no corresponding density step.

The lower two panels of Figure 3 shows where all cells in each model lies on a phase diagram. Also plotted are contours of heating and cooling rate and the stability curve where the heating and cooling rates are equal. The left hand panel shows the familiar behavior of a simulation with a 'normal' $\mathrm{S}$ curve with just one unstable zone. The black crosses show the location of cells in the midplane, and as expected they all lie at the same point; the last stable point on the lower temperature branch. There is then a vertical line of cells showing cells heating up at constant pressure just above the midplane. Adiabatic cooling (and sometimes heating) explains why cells do not always lie on the stability curve.

In contrast, in the Cloudy run, there is a second cluster of cells around the intermediate stable zone on the stability curve (circled in the lower right panel of Figure 3). These are the dense cells seen on the contour plot. Because the cells are on a stable part of the stability curve, they heat up only slowly since radiative cooling is able to almost balance the heating. An outflow does not form. Once the gas heats up enough to reach the upper limit of the second stable zone, they heat up more quickly; however the start of the upper stable branch occurs at a much lower temperature than in the original simulation (around $6 \times 10^{5} \mathrm{~K}$ vs $3 \times 10^{6} \mathrm{~K}$ ). Since the unstable heating process starts at a higher temperature, there is significantly less energy deposited into the gas, and so the final outflow is much slower and unstable.

In order to see if a stable outflow forms at larger radii, we next extend the simulation domain size. However this requires care, since the accretion disk in an LMXB has to fit within the primary's Roche lobe. An estimate of the maximum disk size in a binary system is provided by the radius of the last non-intersecting particle orbit around the accretor. This is given by

$$
\frac{r_{d}(\max )}{a}=\frac{0.6}{1+q}
$$

where $\mathrm{q}$ is the secondary mass divided by the primary, and a is the separation of the binary system (Warner 2003). Reliable system parameters are available for two black-hole binaries that show clear evidence for disk winds in the soft state (Ponti et al. 2012): GROJ1655-40 and GRS1915+105. Using the data from Ritter \& Kolb (2003), we calculate maximum stable disk radii of $4.7 \times 10^{11}$ and $3.8 \times 10^{12} \mathrm{~cm}$, respectively. Our initial runs with an outer boundary at $2 \mathrm{R}_{\mathrm{IC}}=9.6 \times 10^{11} \mathrm{~cm}$ therefore represent a reasonable maximum disk radius; when extending the domain, we need to modify the simulation so that the disk does not exceed the size of the binary.

In order to achieve this, the initial density in the new expanded runs is set along the midplane according to the equation 5 up to a radius of $2 R_{I C}$. Outside this radius, it is multiplied by a factor of $\exp \left(20\left(1-\left(\mathrm{r} / 2 \mathrm{R}_{\mathrm{IC}}\right)\right)\right)$ to produce a steep but not instant dropoff to the simulation minimum density of $1 \times 10^{-22} \mathrm{~g} \mathrm{~cm}^{-3}$

The density at the midplane up to $r=2 R_{I C}$ is reset each cycle, but the midplane density at larger radii is allowed to vary, with the same density floor applied as in the rest of the model. The boundary condition varies along the midplane, with the normal reflecting boundary condition set out to a radius of $2 \mathrm{R}_{\mathrm{IC}}$ but an outflow condition set at larger radii. The fate of material flowing 'downwards' through the outer disk is not computed, little mass is actually lost through this boundary so this somewhat unphysical situation does not significantly affect the results. We compute two new runs with these parameters, the parameters and some results are summarised in Table 1. They are labeled "Extended Blondin" and "Extended Cloudy".

Figure 4 shows the density and velocity distribution of the two extended runs. The gas continues to accelerate in both cases, but the extended Cloudy run is still slower than the Blondin version. It is initially surprising to note that the mass loss through the outer boundary in the extended Cloudy run has significantly increased over the smaller domain. The reason is most likely because we do not capture the Mach 1 surface in the small domain, and so the conditions in the larger run can affect the conditions at the base of the wind. This ef- 

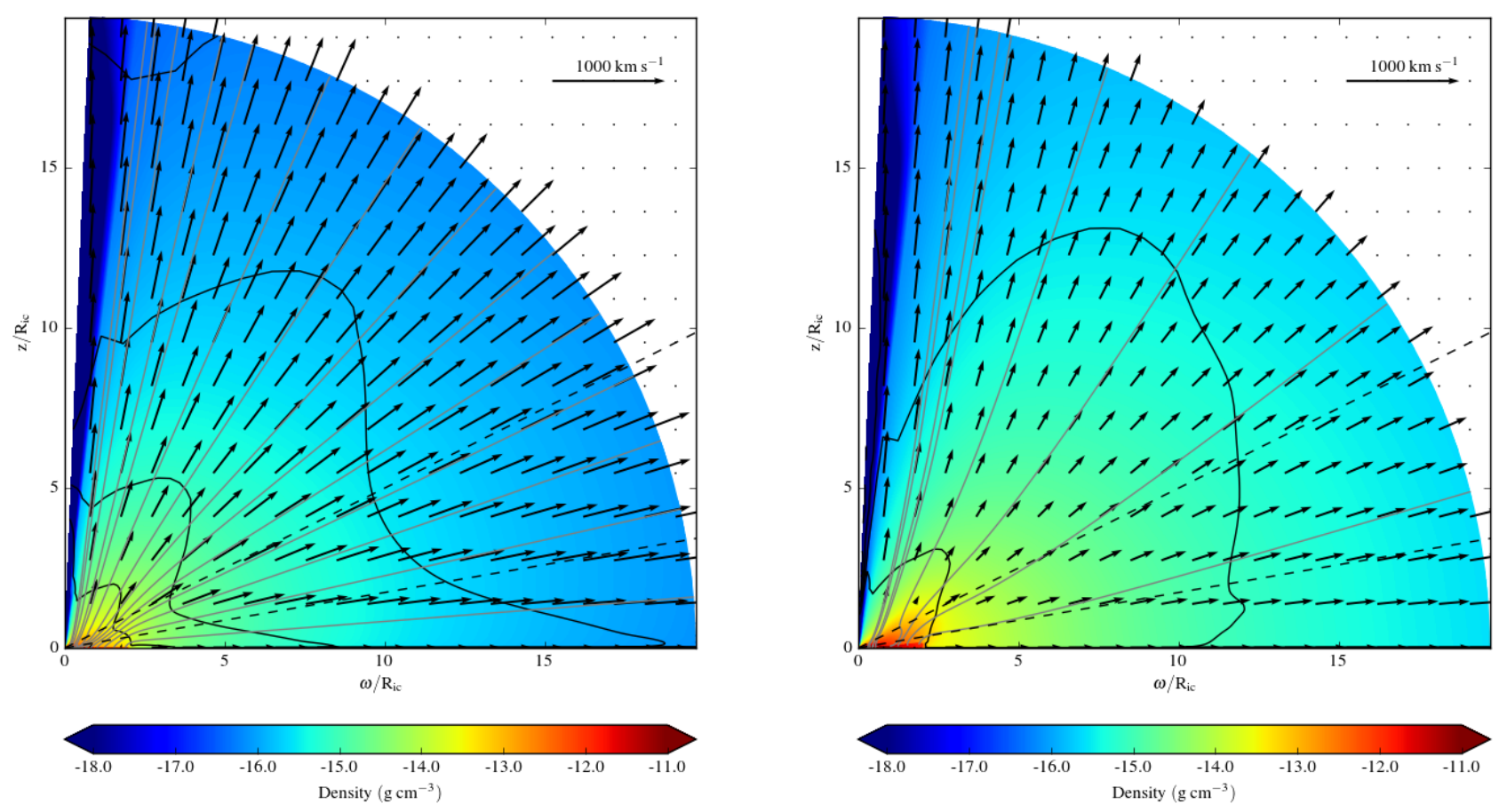

Figure 4. Comparison between two wind geometries with extended simulation domain. The left hand panel shows the extended Blondin simulation, the right hand panel shows the extended Cloudy run. Grey lines show streamlines, and the black line shows the location of the Mach surfaces. In each case, the innermost line (seen inside about $2 \mathrm{R}_{\mathrm{IC}}$ ) is the Mach 1 surface. The two dotted lines show the location of the $60^{\circ}$ and $80^{\circ}$ sightlines.

fect is much less in evidence in the Blondin runs, because we do include most of the Mach 1 surface in the smaller domain. Figure 5 shows only the inner $2 \mathrm{R}_{\mathrm{IC}}$ of the extended Cloudy run, and it is clear that there are differences compared to the simulation conducted in the smaller domain. The mass loss seen in the extended Cloudy run, $15 \dot{\mathrm{M}}_{\mathrm{acc}}$, is of the right order of magnitude to cause instabilities in the disk and perhaps cause state change (Shields et al. 1986).

\section{DISCUSSION}

Whilst the overall mass loss rate is significant, it is important to ask whether this wind would be observable. All XRBs identified as having outflows exhibit FeXXVI absorption (Ponti et al. 2012) Some also have FeXXV lines and

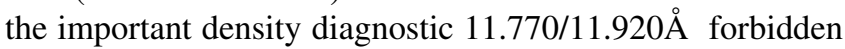
lines of FeXXII are seen in at least one system (Miller et al. 2006). The typical Hydrogen column density derived from photoionization modelling of lines seen in outflows is between $5 \times 10^{20}$ and $10^{24} \mathrm{~cm}^{-2}$ (Díaz Trigo \& Boirin 2016). The ionization parameter calculated from the same modelling is in the range $\log (\xi) \sim 1.8-6$ (Díaz Trigo \& Boirin 2013) with most systems characterised by $\log \xi \geq 3$

Figure 6 shows the value of $\xi$ calculated in each cell in the extended Cloudy model (still in the optically thin limit) and it confirms that almost the entire model contains gas with an ionization parameter in the range $3<\log (\xi)<4$, in the middle of the range of observed ionization parameters.

Figure 7 shows the column density through the model for
Hydrogen and the two of the observed Iron species. We see immediately that the model is somewhat Compton thick for angles greater than about $84^{\circ}$. Almost all sightlines have a Hydrogen column density in the observed range, with more equatorial sightlines matching the higher estimates.

Figure 7 also shows the column density of these three iron species through the model. The relative abundances of these ions is computed using the same lookup table method as we used to calculate the heating and cooling rates. The same thin shell CLOUDY model is used to produce these abundance tables. In the optically thin limit, an estimate of the equivalent width of lines can be obtained from (Matt 1994)

$$
E W=18.1\left(\frac{A_{F e}}{3.3 \times 10^{-5}}\right)\left(\frac{N_{H}}{10^{22} \mathrm{~cm}^{-2}}\right)\left(\frac{f_{l u}}{0.5}\right) f_{\text {ion }} \mathrm{eV} .
$$

where $A_{F e}$ is the iron abundance relative to Hydrogen. Treating the Iron $K \alpha$ absorption doublet as a single line with oscillator strength $f_{l u}=0.46$ and assuming all ions are in the lower level we can estimate the equivalent width of this line as seen from different angles. This is plotted on figure 7 as the dashed curve. Where outflows are detected, they are typically at the level of $10 \mathrm{eV}$ and greater Ponti et al. (2012) so based upon this simple estimate, we would expect this wind to be detectable for viewing angles of about $50^{\circ}$ and greater.

Whilst this simple calculation demonstrates that that the simulation produces a gas distribution that would be detectable, at least in the Iron $\mathrm{K} \alpha$ absorption line, it does not 


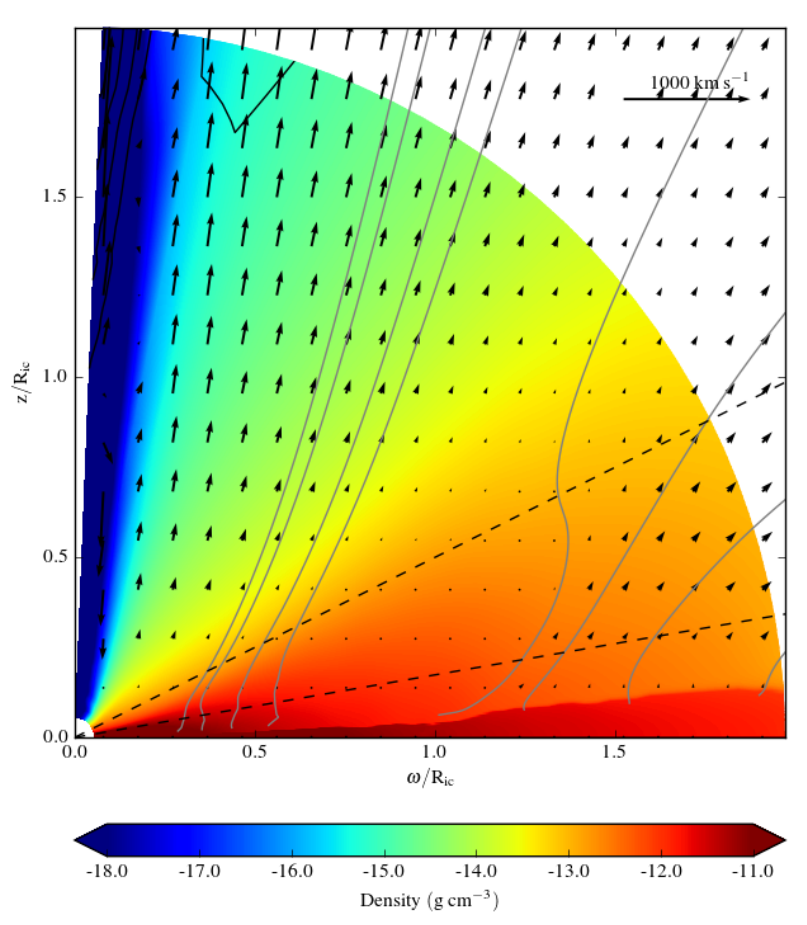

Figure 5. Inner $2 \mathrm{R}_{\mathrm{IC}}$ of the extended domain simulation using CLOUDY heating and cooling rates

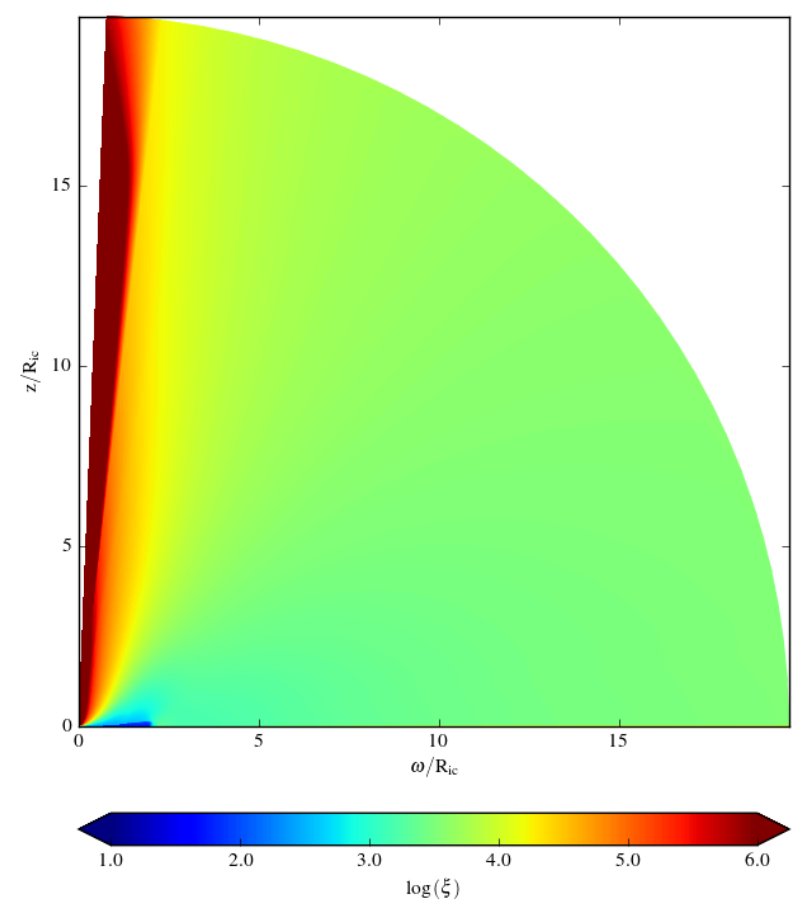

Figure 6. Ionization parameter recorded in extended cloudy model

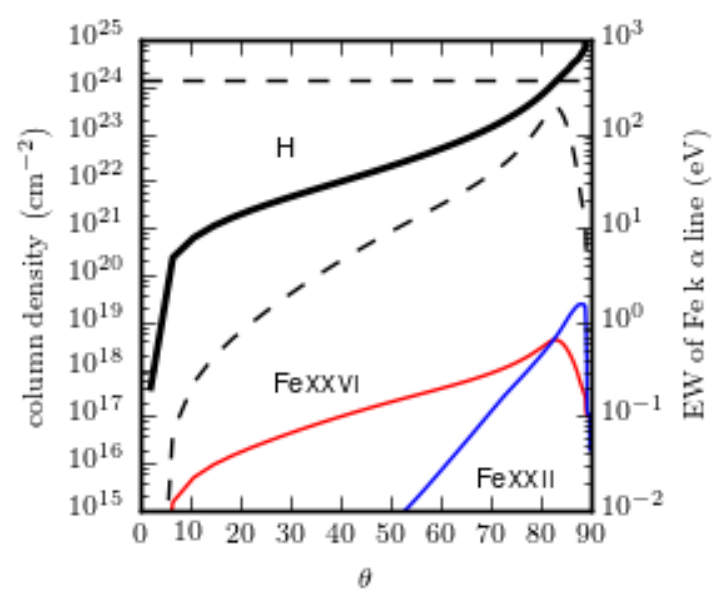

Figure 7. Column density of Hydrogen and two Iron species through the extended cloudy model. The horizontal dashed line shows the value of $1 / \sigma_{\mathrm{T}}$, and the dashed curve (plotted on the right hand axis) shows the predicted equivent width of an Iron $\mathrm{K} \alpha$ absorption feature

demonstrate that it would produce the blue-shifted features that suggest outflows. Indeed the velocity profiles shown in figures 3-5 show that the typical velocities in the Cloudy models are $100-200 \mathrm{kms}^{-1}$ with the dense parts of the wind $\left(\mathrm{n}_{\mathrm{H}}>1 \times 10^{12} \mathrm{~cm}^{-3}\right)$ showing outflow velocities of less than $100 \mathrm{kms}^{-1}$. Typical velocities seen in LMXB outflows are between 400 and $3000 \mathrm{kms}^{-1}$ (Díaz Trigo \& Boirin 2016), so the wind produced here is certainly at the lower end of observed velocities. The production of detailed synthetic spectra is beyond the scope of this work. However, we can compute a simple line profile for the Iron $\mathrm{K} \alpha$ absorption feature that we have just demonstrated is likely to be visible.

The absorption coefficient for a spectral line, neglecting collisional de-excitation is given by

$$
\alpha(\nu)=\frac{\pi e^{2}}{m_{e} c} \phi(\nu) n_{l} f_{l u},
$$

where $\mathrm{n}_{\mathrm{l}}$ is the number density of the lower ion and $\phi \nu$ is the line profile function, which we treat as a thermally broadened Gaussian. For each radial cell, we compute the line opacity as a function of frequency, Doppler shifted to the radial velocity of that cell. We then compute a total, frequency dependent optical depth for a given sightline by performing a summation over each cell $\mathrm{i}$, with absorption coefficient $\alpha_{\mathrm{i}}(\nu)$ and radial extent $\Delta r_{i}$ in that sightline;

$$
\tau(\nu)=\sum_{i=\text { inner }}^{i=\text { outer }} \alpha_{i}(\nu) \Delta r_{i}
$$

We then produce a synthetic line profile using

$$
F(\nu)=e^{-\tau(\nu)}
$$

Figure 8 shows the resulting line profiles for two viewing angles. We see that there are two features at each angle. There are black absorption features at the rest velocity, somewhat narrower at $60^{\circ}$ than at $80^{\circ}$. This is produced by material at small radii, where the gas is dense but slow moving. The fact that the lines are saturated means that strictly speak- 


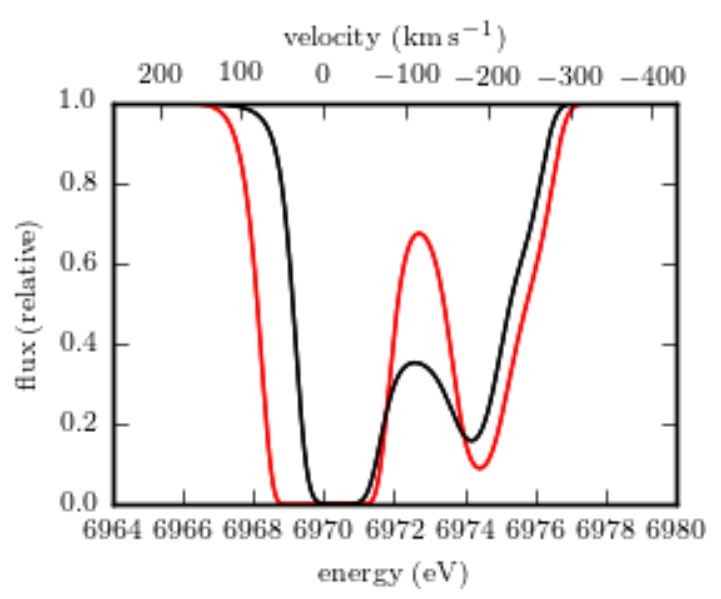

Figure 8. Synthetic Iron $\mathrm{K} \alpha$ lines at $60^{\circ}$ (black) and $80^{\circ}$ (red) for the extended Cloudy simulation

ing Equation 9 is not universally applicable in the model; We would expect the EW to reach a maximum value as the lines saturate. Nonetheless, the statement that the wind should produce observable lines is still reasonable although the actual values of EW shown on Figure 7 should be taken as upper limits. The figures in Table 1 for the radial velocities of the high density plasma in the simulations underline the fact that where the gas is dense, it is slow moving. There are only two observed systems where densities of the absorbing gas have been estimated (Schulz et al. 2008; Kallman et al. 2009) and in those cases fast moving (around $400 \mathrm{~km} \mathrm{~s}^{-1}$ ) gas with number densities of $10^{13}-10^{15} \mathrm{~cm}^{-3}$ was observed. Our maximum radial blue shifted velocities for gas with density above $10^{12} \mathrm{~cm}^{-3}$ are less than $100 \mathrm{~km} \mathrm{~s}^{-1}$ in all cases, slower in the two Cloudy runs than in the Blondin runs. There is a second feature at about $200 \mathrm{~km} \mathrm{~s}^{-1}$ produced by the more tenuous accelerated gas at larger radii. This underlines the fact that this outflow is significantly slower than most reported in the literature. It is interesting to note, however, that the mass loss rate is still significant, confirming the results of Luketic et al. (2010) and HP15.

Our next project is to compute the heating and cooling rates using a full treatment of radiative transfer (RT). However, it is instructive to calculate whether it is likely that this will have an effect. We can assess this by checking whether the plasma at the base of the wind is optically thin to its own line radiation. This is important, since line cooling is the dominant cooling mechanism at the base of the wind. If the line radiation is unable to escape, then the thermal balance will change significantly.

The optical depth of a spectral line in an expanding spherical atmosphere is given by (Castor et al. 1975)

$$
\tau_{L}=\kappa_{L} \rho v_{t h}\left|\frac{d v}{d r}\right|^{-1},
$$

where $v_{\text {th }}$ is the thermal velocity of the gas, and the final term is the velocity gradient. This equation is a form of the Sobolev approximation. $\kappa_{L}$ is the monochromatic line absorption coefficient, per unit mass, divided by the line profile function $\left(\Delta v_{D}=\nu_{0} v_{t} h / c\right)$,

$$
\kappa_{L}=\frac{\pi e^{2}}{m c} g_{1} f\left(\frac{n_{1}}{g_{1}}-\frac{n_{2}}{g_{2}}\right) \frac{1}{\Delta v_{D}} \frac{1}{\rho}
$$

Substituting for $\kappa_{L}$, assuming all ions are in the ground state $\left(n_{2}=0\right)$ and simplifying we obtain

$$
\tau_{L}=\frac{\pi e^{2}}{m c} n_{1} f \frac{c}{\nu_{0}}\left|\frac{d v}{d r}\right|^{-1} .
$$

Line cooling dominates the cooling rate at the midplane of the simulation, and the main cooling line for $\xi=\xi_{\text {cool,max }}=15$ and $\mathrm{T}=\mathrm{T}_{\text {cool, } \max }=4.3 \times 10^{4} \mathrm{~K}$ is O VI $\lambda 1032 / 1038 \AA$, making up about $30 \%$ of the total cooling rate. Taking this doublet as an example, we can attempt to quantify the effect of line optical depth on the cooling rate. The oscillator strengths are $\mathrm{f}=0.13,0.07$ respectively, we treat them as a single line with $\mathrm{f}=0.2$. At $\mathrm{r}=\mathrm{R}_{\mathrm{IC}}, n_{1}=\mathrm{n}_{\mathrm{OVI}}=6.7 \times 10^{8} \mathrm{~cm}^{-3}$, the vertical velocity gradient at the midplane is very variable, we take a representative value of $\mathrm{dv}_{\mathrm{z}} / \mathrm{dz}=2 \times 10^{-4} \mathrm{~s}^{-1}$ (the radial velocity gradient is zero by construction at the midplane, and the Keplerian velocity shear is an order of magnitude lower than the vertical velocity gradient). These values give an optical depth of about $2 \times 10^{5}$, highly optically thick.

The effect of this is complex. The line cooling will likely be suppressed, however even in a region with such a large optical depth, line radiation can still escape after many scatters. The degree to which line cooling is suppressed depends on the chance of an ion collisionally de-exciting during the scattering process. The effect of this is to reduce the line cooling rate by a factor of $\mathrm{A}_{21} / \tau \mathrm{n}_{\mathrm{e}} \mathrm{q}_{21}$ when the optical depth is large (Raymond 1993, and private communication). $\mathrm{A}_{21}$ is the Einstein radiative recombination rate and $\mathrm{q}_{21}$ is the collisional de-excitation rate. For the $\mathrm{O}$ VI line, this gives a 45 fold suppression factor at $r=R_{I C}$. Although this factor decreases with radius there will certainly be an effect on the wind solution; HP15 experimented with suppressing line cooling by a factor of 5, and this (together with a four-fold increase in $\mathrm{X}$-ray heating) significantly increased the mass outflow rate and velocity of the wind. Naturally, the any changes to the flow will modify the density, temperature and therefore the suppression factor which will in turn modify the flow. The forthcoming full RT simulations will capture this behavior.

\section{CONCLUSIONS}

HP15 demonstrated that the heating and cooling rates are critical in defining the velocity and density of thermal winds in LMXBs, as calculated in hydrodynamical simulations. Modest changes to those rates produced winds with velocities approaching those seen in observations of LMXBs. The changes made to the rates were physically motivated but they were not calculated from the illuminating SED, plasma composition or any other physical properties of the systems.

In this study, we replaced the parametrized heating and cooling curves used by HP15 with more realistic heating and cooling curves, as calculated with CLOUDY. In our hydrodynamical simulations using these rates, the resulting outflow is slower $\left(\mathrm{v} \lesssim 200 \mathrm{~km} \mathrm{~s}^{-1}\right)$ than in the simulations carried out 
by HP15. It is also slower than the wind velocities typically observed in LMXBs, which lie between 400 and $3000 \mathrm{kms}^{-1}$ (Díaz Trigo \& Boirin 2016), However, the overall mass-loss rate of the outflow is significant $\left(\dot{M}_{\text {wind }} \simeq 15 \dot{M}_{a c c}\right)$ and may be enough to destabilize the disk and perhaps prompt a state change.

In addition, the wind arises at rather large distances from the central object, at least an order of magnitude greater than the $0.1 \mathrm{R}_{\mathrm{IC}}$ seen in previous studies (e.g. Begelman et al. 1983; Woods et al. 1996; Proga \& Kallman 2002). This is because gas in the new simulations heats up more slowly as a result of intermediate stable temperatures existing on the CLOUDY calculated stability curve, meaning only at larger radii does the gas gain sufficient thermal energy to escape the gravitational potential. This is in line with the observation that outflowing material in LMXBs is only seen in long period systems (Díaz Trigo \& Boirin 2016), although Miller et al. (2006) found that the outflowing material in GRO J1655-40 was best modelled by an absorber at about $0.001 R_{I C}$.

\section{FUTURE WORK}

The simulations presented here are all calculated in the optically thin limit. In other words the calculation of the ionization parameter used to compute the heating and cooling rates in each cell takes no account of absorption between the central X-ray source and the cell in question. Since heating takes place in all cells, this is incorrect in detail. In fact, we have shown that the cells closest to the disk in our simulation are likely to be optically thick to their own line cooling radiation, and so our next step is to use a full RT code to compute the heating and cooling rates in each cell, thereby taking account of all absorption processes. This is a significantly more complex undertaking and will be described in a following paper.

Calculations in this work made use of the Iridis4 Supercomputer at the University of Southampton. DP acknowledges support provided by NASA under ATP grant NNX14AK44G. NH and CK acknowledge support by the Science and Technology Facilities Council grant ST/M001326/1. KSL acknowledges the support of NASA for this work through grant NNG15PP48P to serve as a science adviser to the Astro-H project We would also like to thank Stuart Sim and James Matthews for many helpful suggestions to improve this work. Finally we thank the referee John Raymond for a very useful review.

Software: ZEUS (Stone \& Norman 1992), CLOUDY (Ferland et al. 2013)

\section{REFERENCES}

Begelman, M. C., McKee, C. F., \& Shields, G. A. 1983, ApJ, 271, 70

Blandford, R. D., \& Payne, D. G. 1982, MNRAS, 199, 883

Blondin, J. M. 1994, ApJ, 435, 756

Blondin, J. M., Kallman, T. R., Fryxell, B. A., \& Taam, R. E. 1990, ApJ, 356, 591

Castor, J. I., Abbott, D. C., \& Klein, R. I. 1975, ApJ, 195, 157

Chakravorty, S., Petrucci, P.-O., Ferreira, J., et al. 2016, A\&A, 589, A119

Díaz Trigo, M., \& Boirin, L. 2013, Acta Polytechnica, 53, 659

—. 2016, Astronomische Nachrichten, 337, 368

Dyda, S., Dannen, R., Waters, T., \& Proga, D. 2016, ArXiv e-prints, arXiv:1610.04292 [astro-ph.HE]

Emmering, R. T., Blandford, R. D., \& Shlosman, I. 1992, ApJ, 385, 460

Fender, R. P., Belloni, T. M., \& Gallo, E. 2004, MNRAS, 355, 1105

Ferland, G. J., Porter, R. L., van Hoof, P. A. M., et al. 2013, RMxAA, 49, 137

Higginbottom, N., \& Proga, D. 2015, ApJ, 807, 107

Icke, V. 1980, AJ, 85, 329

Jimenez-Garate, M. A., Raymond, J. C., Liedahl, D. A., \& Hailey, C. J. 2001, ApJ, 558, 448

Kallman, T. R., Bautista, M. A., Goriely, S., et al. 2009, ApJ, 701, 865

King, A. L., Miller, J. M., Raymond, J., et al. 2012, ApJL, 746, L20

Luketic, S., Proga, D., Kallman, T. R., Raymond, J. C., \& Miller, J. M. 2010, ApJ, 719, 515
Matt, G. 1994, MNRAS, 267, L17

Miller, J. M., Raymond, J., Fabian, A., et al. 2006, Nature, 441, 953

Nayakshin, S., Kazanas, D., \& Kallman, T. R. 2000, ApJ, 537, 833

Ponti, G., Fender, R. P., Begelman, M. C., et al. 2012, MNRAS, 422, 11

Proga, D., \& Kallman, T. R. 2002, ApJ, 565, 455

Proga, D., Stone, J. M., \& Kallman, T. R. 2000, ApJ, 543, 686

Raymond, J. C. 1993, ApJ, 412, 267

Ritter, H., \& Kolb, U. 2003, A\&A, 404, 301

Schulz, N. S., Kallman, T. E., Galloway, D. K., \& Brandt, W. N. 2008, ApJ, 672,1091

Shakura, N. I., \& Sunyaev, R. A. 1973, A\&A, 24, 337

Shields, G. A., McKee, C. F., Lin, D. N. C., \& Begelman, M. C. 1986, ApJ, 306, 90

Shlosman, I., \& Vitello, P. 1993, ApJ, 409, 372

Shlosman, I., Vitello, P. A., \& Shaviv, G. 1985, ApJ, 294, 96

Stone, J. M., \& Norman, M. L. 1992, ApJS, 80, 753

Warner, B. 2003, Cataclysmic Variable Stars

Woods, D. T., Klein, R. I., Castor, J. I., McKee, C. F., \& Bell, J. B. 1996, ApJ, 461, 767 